International Electronic Journal of Algebra

VOLUME 21 (2017) 180-197

\title{
WEAKLY NIL-CLEAN INDEX AND UNIQUELY WEAKLY NIL-CLEAN RINGS
}

\author{
Andrada Cîmpean and Peter Danchev \\ Received: 09 July 2016; Revised: 05 September 2016 \\ Communicated by Huanyin Chen
}

\begin{abstract}
We introduce and study the weakly nil-clean index associated to a ring. We also give some simple properties of this index and show that rings with the weakly nil-clean index 1 are precisely those rings that are abelian weakly nil-clean, thus showing that they coincide with uniquely weakly nil-clean rings. Next, we define certain types of nilpotent elements and weakly nil-clean decompositions by obtaining some results when the weakly nil-clean index is at most 2 and, moreover, we somewhat characterize rings with weakly nil-clean index 2. After that, we compute the weakly nil-clean index for $\mathbb{T}_{2}\left(\mathbb{Z}_{p}\right), \mathbb{T}_{3}\left(\mathbb{Z}_{p}\right)$ and $\mathbb{M}_{2}\left(\mathbb{Z}_{3}\right)$, respectively, as well as we establish a result on the weakly nilclean index of $\mathbb{M}_{n}(R)$ whenever $R$ is a ring. Our results considerably extend and correct the corresponding ones from [Int. Electron. J. Algebra 15(2014), 145-156].
\end{abstract}

Mathematics Subject Classification (2010): 16E50, 16S34, 16U10

Keywords: Nil-clean ring, uniquely nil-clean ring, weakly nil-clean ring, uniquely weakly nil-clean ring, abelian ring

\section{Introduction and background}

All rings $R$ in this paper are associative with 1, but not necessarily commutative. The symbols $U(R), J(R), I d(R)$, and $N i l(R)$ will stand for the group of units, the Jacobson radical, the set of idempotents and the set of nilpotents of $R$, respectively. Also, for $e \in R$, we define $C(e)=\{x \in R \mid e x=x e\}$. All other unexplained explicitly below notion and notation are standard and follow essentially those from [9]. For instance, $\mathbb{M}_{n}(R)$ denotes the full $n \times n$ matrix ring and $\mathbb{T}_{n}(R)$ denotes the upper triangular $n \times n$ matrix ring.

In [8] a ring $R$ is said to be nil-clean if each element $a \in R$ can be represented as $a=b+e$, where $b \in \operatorname{Nil}(R)$ and $e \in I d(R)$; note that this is equivalent to the presentation that, for every $a \in R$, we have $a=b-e$. If this presentation is unique, the ring $R$ is called uniquely nil-clean. This is tantamount to the requirement that the existing idempotent $e$ is unique (see, e.g., $[5,8]$ ). 
On the other vein, in [3] and [7] was stated the definition of a weakly nil-clean ring as such a ring $R$ for which any element $a \in R$ is of the form $a=b+e$ or $a=b-e$, where $b \in \operatorname{Nil}(R)$ and $e \in I d(R)$. Moreover, a ring $R$ is said to be uniquely weakly nil-clean if the existing idempotent $e$ is unique.

This work is motivated by the notions of unique nil-cleanness and weak nilcleanness and we will combine them into a new concept. So, the aim of the current paper is to explore some variations of unique weak nil-cleanness in order to enlarge the principal known results on unique nil-cleanness from [5] and [6]. Although weakly nil-clean rings were recently completely characterized independently in [6] and [12], the full description of uniquely weakly nil-clean rings remains interesting and worthy of exploration. For any $a \in R$, let $\mathcal{E}(a)=\left\{e \in R \mid e^{2}=e, a-e \in U(R)\right\}$ and then the clean index of $R$, denoted as $c(R)$, is defined in [10] by $c(R)=$ $\sup \{|\mathcal{E}(a)|: a \in R\}$. For any $a \in R$, set $\eta(a)=\left\{e \in R \mid e^{2}=e\right.$ and $a-e \in$ $\operatorname{Nil}(R)\}$ and then the nil-clean index of $R$, denoted as $N i n(R)$, is defined in [1] by $\sup \{|\eta(a)|: a \in R\}$. In this way, for a more comprehensive investigation of these two notions and, especially, as a natural generalization of the nil-clean index, we also define the concept of weakly nil-clean index of a ring. Thereby, as it will be showed below, a ring is uniquely weakly nil-clean if and only if it is weakly nil-clean of weakly nil-clean index 1 .

The paper is organized as follows: In the first section, we already have given the main definitions of the used concepts. In the second section, we set and explore in details the weakly nil-clean index of rings and discuss the original notion of uniquely weakly nil-clean rings stated in Problem 3 of [7]. We also investigate here some other aspects of unique weak nil-cleanness which arise from its specific definition. And we close the work in the final third section by stating certain open problems of some interest and importance.

\section{Weakly nil-clean index of rings}

In [10] and [11] the clean index $c(R)$ of a ring $R$ was defined and studied. Imitating this, in [1] was introduced the nil-clean index $N \operatorname{in}(R)$ of $R$ and a detailed study was given.

In parallel to these two notions, we proceed by stating the following concepts.

Definition 2.1. Let $R$ be a ring and $a \in R$. We define the set

$$
\alpha(a)=\left\{e \in R: e^{2}=e \text { and } a-e \text { or } a+e \text { is a nilpotent }\right\} .
$$


Definition 2.2. For an element $a \in R$ the weakly nil-clean index of $a$, abbreviated as $w n c(a)$, is defined to be the cardinality of the set $\alpha(a)$.

Definition 2.3. We define the weakly nil-clean index of a ring $R$ as follows:

$$
w n c(R)=\sup \{|\alpha(a)|: a \in R\} .
$$

We foremost start with a series of elementary but useful basic properties of the operator $w n c(R)$ which extend the analogous ones in [1].

Lemma 2.4. For any ring $R$ the inequality $w n c(R) \geq 1$ holds. In addition, if $R$ is a ring which has at most $n$ idempotents or at most $n$ nilpotents, then $w n c(R) \leq n$.

Proof. Straightforward.

Example 2.5. A direct check shows that wnc $\left(\mathbb{Z}_{3}\right)=1$.

Lemma 2.6. If $R$ is a ring with a subring $S$, then $w n c(R) \geq w n c(S)$.

Proof. Follows in the same manner as [1, Lemma 2.2].

Lemma 2.7. If $R$ is a ring with a nil ideal $I$, then $w n c(R / I) \leq w n c(R)$.

Proof. Letting $a \in R$ be an arbitrary element, then for any idempotent $b+I \in$ $\alpha(a+I)$, so $b^{2}-b \in I$ and there exists $e \in I d(R)$ with $b+I=e+I$, one may derive that $(a+I)-(b+I)=(a-e)+I$ with $(a-e)^{t} \in I$ or that $(a+I)+(b+I)=(a+e)+I$ with $(a+e)^{t} \in I$ for some $t \in \mathbb{N}$. Since $I$ is nil, it follows that either $a-e \in N i l(R)$ or $a+e \in \operatorname{Nil}(R)$. Consequently, $e \in \alpha(a)$ and thus $|\alpha(a)| \geq|\alpha(a+I)|$, as needed.

Remark 2.8. In [1, Lemma 2.4 (1)] the condition "If idempotents lift modulo $I$ " is redundant, because I is a nil-ideal. Moreover, the inequality $\operatorname{Nin}(R / I) \geq N i n(R)$ is not true and the purported there proof is errorneous. This can be subsumed via the following construction: set $R=\mathbb{Z}_{p}$ and $I=\left\{\left(a_{i j}\right) \in \mathbb{T}_{n}(R): \forall a_{i i}=0\right\}$. It is readily seen that this is a nil-ideal of $\mathbb{T}_{n}(R)$ with the property that $\mathbb{T}_{n}(R) / I \cong$ $R \times \cdots \times R$, where the product is taken $n$ times.

Next, choosing $n=2=p$, we detect that $\mathbb{T}_{2}\left(\mathbb{Z}_{2}\right) / I \cong \mathbb{Z}_{2} \times \mathbb{Z}_{2}$, whence with the aid of $\left[1\right.$, Lemma 2.3] we derive $\operatorname{Nin}\left(\mathbb{T}_{2}\left(\mathbb{Z}_{2}\right) / I\right)=\operatorname{Nin}\left(\mathbb{Z}_{2} \times \mathbb{Z}_{2}\right)=\operatorname{Nin}\left(\mathbb{Z}_{2}\right) \operatorname{Nin}\left(\mathbb{Z}_{2}\right)=$ $1 \cdot 1=1$. On the other hand, $\left[8\right.$, Theorem 4.1] is a guarantor that $\mathbb{T}_{2}\left(\mathbb{Z}_{2}\right)$ is nil-clean, so that $\operatorname{Nin}\left(\mathbb{T}_{2}\left(\mathbb{Z}_{2}\right)\right)=$ wnc $\left(\mathbb{T}_{2}\left(\mathbb{Z}_{2}\right)\right)=2$, owing to Example 2.23 listed below. Thus this contradiction demonstrates $\operatorname{Nin}(R / I)<N i n(R)$.

If now we choose $n=3=p$, then the same trick successfully works to manifestly illustrate with the help of Example 2.24 quoted below that wnc $(R)>w n c(R / I)$. 
The next assertion improves [1, Lemma 2.8].

Lemma 2.9. For any ring $R$ the inequalities $c(R) \geq w n c(R) \geq N$ in $(R)$ hold.

Proof. Since the second inequality is trivial, we will deal only with the first one. To that goal, for any $a \in R$, writing that $a=q+e$ or $a=q-e$ for some nilpotent $q$ and idempotent $e$, we deduce that $a+1=(q+1)+e$ with a unit $q+1$ or that $a=(q-1)+(1-e)$ with a unit $q-1$, so that both $a+1$ and $a$ are clean elements. Since $a+1 \in R$, the further proof goes on as in [1, Lemma 2.8].

Remark 2.10. Note that if $R$ is a nil-clean ring, then $w n c(R)=N i n(R)$.

The next assertion extends [1, Theorem 3.2].

Proposition 2.11. Suppose $R$ is a ring. Then $w n c(R)=1$ if and only if $R$ is abelian.

Proof. First of all we will prove that $w n c(R)=1$ if and only if $R$ is abelian and for any non-zero idempotent $e \in R$, the relation $e \neq m+n$ holds for all $m, n \in \operatorname{Nil}(R)$.

Since with Lemma 2.9 at hand we have $1=w n c(R) \geq N \operatorname{in}(R) \geq 1$, it follows that $\operatorname{Nin}(R)=1$ and by [1, Lemma 3.1] we get that $R$ is abelian and for any idempotent $0 \neq e \in R$, the ratio $e \neq m+n$ is valid for all $m, n \in N i l(R)$.

Now let $R$ be abelian and, for any idempotent $e \in R \backslash\{0\}$, the inequality $e \neq m+n$ is true for all $m, n \in N i l(R)$. Suppose, for concreteness, $a \in R$ has two weakly nil-clean decompositions. We have three possible cases:

(1) $a=e_{1}+n_{1}=e_{2}+n_{2}$, with $e_{1}$ and $e_{2}$ idempotents and $n_{1}, n_{2} \in \operatorname{Nil}(R)$. In this case the decompositions are actually nil-clean, so this situation was handled in [1, Lemma 3.1] and leaded to $e_{1}=e_{2}$. It follows now that $w n c(R)=1$.

(2) $a=-e_{1}+n_{1}=-e_{2}+n_{2}$, with $e_{1}$ and $e_{2}$ idempotents and $n_{1}, n_{2} \in \operatorname{Nil}(R)$. Then $-e_{1}\left(1-e_{1}\right)+n_{1}\left(1-e_{1}\right)=-e_{2}\left(1-e_{1}\right)+n_{2}\left(1-e_{1}\right)$, so $e_{2}\left(1-e_{1}\right)=n_{2}(1-$ $\left.e_{1}\right)-n_{1}\left(1-e_{1}\right)$. Since $R$ is abelian, the element $e_{2}\left(1-e_{1}\right)$ is an idempotent and both $n_{2}\left(1-e_{1}\right), n_{1}\left(1-e_{1}\right)$ are nilpotents. So, by hypothesis, we get $e_{2}\left(1-e_{1}\right)=0$, that is $e_{2}=e_{1} e_{2}$. Consequently, $n_{1}-n_{2}=e_{1}-e_{2}=e_{1}\left(1-e_{2}\right)$, and hence by hypothesis we derive that $e_{1}\left(1-e_{2}\right)=0$. Thus $e_{1}=e_{1} e_{2}$ and $e_{1}=e_{2}$. It again follows that $w n c(R)=1$.

(3) $a=-e_{1}+n_{1}=e_{2}+n_{2}$, with $e_{1}$ and $e_{2}$ idempotents and $n_{1}, n_{2} \in \operatorname{Nil}(R)$. Then $e_{2}\left(1-e_{2}\right)+n_{2}\left(1-e_{2}\right)=-e_{1}\left(1-e_{2}\right)+n_{1}\left(1-e_{2}\right)$ and so $e_{1}\left(1-e_{2}\right)=$ $n_{1}\left(1-e_{2}\right)-n_{2}\left(1-e_{2}\right)$. Thus $e_{1}\left(1-e_{2}\right)=0$, i.e., $e_{1}=e_{1} e_{2}$. 
Let $n_{1}^{m}=0$ and $f=e_{1}+e_{2}$. Now lifting $f+n_{2}=n_{1}$ to the $m-t h$ power, we obtain that $\sum_{k=0}^{m}\left(\begin{array}{c}m \\ k\end{array}\right) f^{m-k} n_{2}^{k}=0$. But $f^{k}=\left(e_{1}+e_{2}\right)^{k}=$ $\sum_{l=0}^{m}\left(\begin{array}{c}m \\ k\end{array}\right) e_{1}^{k-l} e_{2}^{k}=e_{1}+e_{2}+e_{1} e_{2}\left(2^{k}-2\right)=e_{1}+e_{2}+e_{1}\left(2^{k}-2\right)=\left(2^{k}-1\right) e_{1}+e_{2}$, so $\sum_{k=0}^{m}\left(\begin{array}{c}m \\ k\end{array}\right)\left(\left(2^{m-k}-1\right) e_{1}+e_{2}\right) n_{2}^{k}=0$, which gives $e_{1} \sum_{k=0}^{m}\left(\begin{array}{c}m \\ k\end{array}\right) 2^{m-k} n_{2}^{k}+$ $\left(e_{2}-e_{1}\right) \sum_{k=0}^{m}\left(\begin{array}{c}m \\ k\end{array}\right) n_{2}^{k}=0$. This is equivalent to $e_{1}\left(2+n_{2}\right)^{k}+\left(e_{2}-e_{1}\right)\left(n_{2}+\right.$ $1)^{k}=0$. Hence $e_{2}\left(n_{2}+1\right)^{k}+e_{1}\left(\left(2+n_{2}\right)^{k}-\left(1+n_{2}\right)^{k}\right)=0$. Multiplying by $\left(1-e_{1}\right)$ we get $\left(1-e_{1}\right) e_{2}\left(n_{2}+1\right)^{k}=0$, but $n_{2}+1$ is a unit, so $e_{2}=e_{1} e_{2}$ and from $e_{1}=e_{1} e_{2}$ we have $e_{1}=e_{2}$. It follows once again that $w n c(R)=1$.

Knowing that $w n c(R)=1$ if and only if $R$ is abelian and for any non-zero idempotent $e \in R$, the relation $e \neq m+n$ holds for all $m, n \in N i l(R)$ and by Lemma 3.1 from [1], we infer that $w n c(R)=1$ if and only if $\operatorname{Nin}(R)=1$ and now using Theorem 3.2 from [1] we get the desired result.

We will now consider the special case of rings having the weakly nil-clean index one and shall completely characterize them. Notice once again that weakly nil-clean rings are independently classified in [6] and [12], respectively. So, we come now to one of our basic statements which does not follow directly by the cited result.

Theorem 2.12. The following are equivalent for a ring $R$ :

(1) $R$ is uniquely weakly nil-clean;

(2) $R$ is abelian weakly nil-clean;

(3) $R \cong R_{1} \times R_{2}$, where $R_{1}$ is either 0 or an abelian nil-clean ring with $J\left(R_{1}\right)$ nil and $R_{1} / J\left(R_{1}\right) \cong B$, where $B$ is a Boolean ring, and $R_{2}$ is either 0 or a local weakly nil-clean ring such that $J\left(R_{2}\right)$ is nil and $R_{2} / J\left(R_{2}\right) \cong \mathbb{Z}_{3}$.

Proof. $(1) \Leftrightarrow(2)$ This is a direct consequence of Proposition 2.11.

$(2) \Leftrightarrow(3)$ It follows directly from [3].

We recall from [5, Theorem 5.4] that a ring $R$ is uniquely nil-clean if and only if $R$ is abelian nil-clean. So, with Theorem 2.12 at hand, one can deduce the following.

Corollary 2.13. A ring $R$ is uniquely nil-clean if and only if $R$ is uniquely weakly nil-clean and $2 \in J(R)$.

As a connection to strongly $\pi$-regular rings, one may state the following strengthening of results on unique nil-cleanness of rings from [5] and [8].

Corollary 2.14. A ring $R$ is uniquely weakly nil-clean if and only if $R$ is abelian strongly $\pi$-regular such that $R / J(R)$ is isomorphic to either a Boolean ring, or to $\mathbb{Z}_{3}$, or to the direct product of two such rings. 
Proof. It is well known that strongly $\pi$-regular rings $R$ have nil $J(R)$. We therefore employ [3] and Theorem 2.12 to get what we asserted.

Remark 2.15. We shall now explore two various notions of unique weak nilcleanness. At the beginning, if we use the "weak unicity" for a ring $R$, i.e., every element $r \in R$ can be written down in at most one way as a nil-clean element or $-r$ in at most one way as a nil-clean element, then we just obtain uniquely weakly nil-clean rings and vice versa.

However, if we use the "strong unicity" for a ring $R$, i.e., every element $r \in R$ can be written down in a unique way as $n+f$, with $n$ a nilpotent and $f$ or $-f$ an idempotent, then such a ring is either uniquely nil-clean of characteristic 2 or uniquely weakly nil-clean but not nil-clean. This follows because we can write $-1=0+(-1)=(-2)+1$, so if $2 \neq 0$ we have that 2 is not a nilpotent.

Remark 2.16. It is worthwhile noticing that indecomposable rings, and hence local rings, always have weakly nil-clean index one.

Remark 2.17. For any ring $R$ and any $s \in R$, we set $P_{s}=e s(1-e)$ and $P_{s}^{\prime}=$ $(1-e)$ se.

Let now $R$ be a ring and $r \in R$. We then have the following weakly nil-clean decompositions for each idempotent $e$ :

$$
e=e+0=\left(e-P_{r}\right)+P_{r}=\left(e-P_{r}^{\prime}\right)+P_{r}^{\prime}=\left(e+P_{r}\right)-P_{r}=\left(e+P_{r}^{\prime}\right)-P_{r}^{\prime} .
$$

Proposition 2.18. Let $R$ be a ring with $w n c(R) \leq 2$. Then, for any $s \in R$ and for any $e \in \operatorname{Id}(R)$, we have $2 e s(1-e)=0$.

Proof. Let $e \in R$ be an idempotent and let $s \in R$.

If $e$ is central, then $R=C(e)$, so for every $s \in R$ we obtain $e s=s e=e s e$ and, therefore, $e s(1-e)=0$, hence $2 e s(1-e)=0$. If $e$ is not central, then there is $s \notin C(e)$ and so $P_{s} \neq 0$ or $P_{s}^{\prime} \neq 0$. We have $e=e+0=\left(e-P_{s}\right)+P_{s}=\left(e-P_{2 s}\right)+P_{2 s}$ and by $\operatorname{wnc}(R) \leq 2$ and $P_{s} \neq 0$ we get $P_{2 s}=0$ or $P_{2 s}=P_{s}$. If $P_{2 s}=P_{s}$, it follows $e 2 s(1-e)=e s(1-e)$ and thus $e s(1-e)=0$, which is a contradiction because $P_{s} \neq 0$. Consequently, $P_{2 s}=0$, so $2 e s(1-e)=0$.

Remark 2.19. Another proof for Proposition 2.18 is as follows:

Let e be an idempotent. We have

$$
e=e+0=(e+e r(1-e))-e r(1-e)=(e-e r(1-e))+e r(1-e)
$$


thus we get three weakly nil-clean decompositions of e. Therefore,

$$
e=e \pm \operatorname{er}(1-e) \text { or } e+e r(1-e)=e-\operatorname{er}(1-e),
$$

which is equivalent to

$$
\operatorname{er}(1-e)=0 \text { or } 2 \operatorname{er}(1-e)=0 .
$$

Corollary 2.20. Let $R$ be a ring with $w n c(R) \leq 2$. Then, for any $s \in R$ and for any $e \in I d(R)$, we have $2(e s-s e)=0$.

Proof. Utilizing $P_{s}$ as in Proposition 2.18, we obtain that $2 e s(1-e)=0$. Now, considering $P_{s}^{\prime}$, we have $2(1-e) s e=0$ and, therefore, $2 e s=2 e s e=2 s e$, so $2(e s-s e)=0$.

Proposition 2.21. Let $R$ be a ring with wnc $(R) \leq 2$ and $e \in \operatorname{Id}(R)$. Then $|R / C(e)| \leq 2$.

Proof. If we assume the contrary, $|R / C(e)|>2$, then there are two different elements, say $s, t \notin C(e)$, such that $s-t \notin C(e)$. By using Remark 2.19 and $w n c(R) \leq 2$, we differ the following cases:

- $P_{s}=P_{t}$ and $P_{s}^{\prime}=P_{t}^{\prime}$, then $e s(1-e)=e t(1-e)$ and $(1-e) s e=(1-e) t e$, hence $e(s-t)=e(s-t) e$ and $(s-t) e=e(s-t) e$, so $e(s-t)=(s-t) e$, which is a contradiction.

- $P_{s}=0$ and $P_{s}^{\prime}=0$, then $e s=e s e$ and $e s e=s e$, so $e s=s e$, which is a contradiction.

- $P_{s}=0$ and $P_{t}^{\prime}=0$, then since $s$ and $t$ are not in $C(e)$, it follows $P_{s}^{\prime} \neq 0$ and $P_{t} \neq 0$ and $P_{s}^{\prime}=P_{t}$ and by this we get $e(1-e) s e=e e t(1-e)$, so et $(1-e)=0$, which is a contradiction.

- $P_{s}=P_{t}$ and $P_{s}^{\prime}=0$, then $P_{t}^{\prime} \neq 0$, so $P_{s}=P_{t}=P_{t}^{\prime}$, which is a contradiction.

Proposition 2.22. Let $R$ be a ring and $e \in I d(R)$. Then

$$
|R / A(e)| \leq|\alpha(e)|
$$

where $A(e)=\{r \in R \mid \operatorname{er}(1-e)=0\}$.

Proof. Letting $n+1 \leq|R / A(e)|$, then we can find an inclusion

$$
\left\{A(e), r_{1}+A(e), \ldots, r_{n}+A(e)\right\} \subseteq R / A(e) .
$$

So, for any $r_{i}, r_{j}$ such that $i, j \in\{1,2, \ldots, n\}$, we have $r_{i}+A(e) \neq r_{j}+A(e)$ and, therefore, $r_{i}-r_{j} \notin A(e)$. It follows that $P_{r_{i}-r_{j}} \neq 0$. Thus $P_{r_{i}} \neq P_{r_{j}}$. Also, for any 
$r_{i}$, we have $r_{i} \notin A(e)$. Hence $P_{r_{i}} \neq 0$. So the set $\{0\} \cup\left\{P_{r_{i}} \mid i \in\{1,2, \ldots, n\}\right\}$ has $n+1$ elements and since for an idempotent $e$ we get $e=e+0=\left(e-P_{r_{i}}\right)+P_{r_{i}}$ for any $i \in\{1,2, \ldots, n\}$, the desired inequality $|R / A(e)| \leq|\alpha(e)|$ follows, as asserted.

We will now compute $w n c(R)$ for some concrete rings $R$. Specifically, we will show that the following equalities hold.

Example 2.23. $w n c\left(\mathbb{T}_{2}\left(\mathbb{Z}_{p}\right)\right)=p$, where $p$ is a prime number.

Proof. It is a well-known fact that a matrix in $\mathbb{T}_{2}\left(\mathbb{Z}_{p}\right)$ is a nilpotent if and only if it has a zero principal diagonal. We are looking now for idempotents. In fact, $\left(\begin{array}{cc}x_{1} & a \\ \overline{0} & x_{2}\end{array}\right)\left(\begin{array}{cc}x_{1} & a \\ \overline{0} & x_{2}\end{array}\right)=\left(\begin{array}{cc}x_{1}^{2} & a\left(x_{1}+x_{2}\right) \\ \overline{0} & x_{2}^{2}\end{array}\right)$, hence $\left(\begin{array}{cc}x_{1} & a \\ \overline{0} & x_{2}\end{array}\right)=\left(\begin{array}{cc}x_{1}^{2} & a\left(x_{1}+x_{2}\right) \\ \overline{0} & x_{2}^{2}\end{array}\right)$, and thus $x_{1}, x_{2} \in\{\overline{0}, \overline{1}\}$ and $a\left(x_{1}+x_{2}-\overline{1}\right)=\overline{0}$. Each pair $\left(x_{1}, x_{2}\right)$ will give a set of solutions for the problem of idempotent matrices.

- case I : $x_{1}=\overline{0}, x_{2}=\overline{0}$, then $S_{1}=\left\{\left(\begin{array}{cc}\overline{0} & \overline{0} \\ \overline{0} & \overline{0}\end{array}\right)\right\}$;

- case II : $x_{1}=\overline{0}, x_{2}=\overline{1}$, then $S_{2}=\left\{\left(\begin{array}{cc}\overline{0} & \alpha \\ \overline{0} & \overline{1}\end{array}\right), \alpha \in \mathbb{Z}_{p}\right\}$;

- case III : $x_{1}=\overline{0}, x_{2}=\overline{1}$, then $S_{3}=\left\{\left(\begin{array}{cc}\overline{1} & \alpha \\ \overline{0} & \overline{0}\end{array}\right), \alpha \in \mathbb{Z}_{p}\right\}$;

- case IV : $x_{1}=\overline{1}, x_{2}=\overline{1}$, then $S_{4}=\left\{\left(\begin{array}{cc}\overline{1} & \overline{0} \\ \overline{0} & \overline{1}\end{array}\right)\right\}$.

Let $A \in \mathbb{T}_{2}\left(\mathbb{Z}_{p}\right)$. Letting $A-E$ be a nilpotent, where $E$ is an idempotent, then $A$ has the main diagonal of the form of an idempotent diagonal (so it has $\overline{0}$ and/or $\overline{1})$. If $A+E$ is a nilpotent, with $E$ an idempotent, then $A$ has in the main diagonal an element from $\{\overline{0}, \overline{-1}\}$. Therefore, except for $A$ with main zero diagonal, only one of the following can hold: $A+E$ or $A-E$ is a nilpotent, with $E$ an idempotent.

Let $A$ be with $\overline{0}$ or $-\overline{1}$ in the main diagonal. We look for $m$ as big as possible such that $A+E_{1}, \cdots, A+E_{m}$ are nilpotents. Thus $E_{1}, \cdots, E_{m}$ share the same main diagonal, that is, they are in the same $S_{i}$. Hence the problem is reduced to finding the maximum cardinality of $S_{i}, i \in\{1,2,3,4\}$. Also, trying to find out the maximum $r$ such that $A-E_{1}, \cdots, A-E_{r}$ are nilpotents, with $A$ having $\overline{0}$ and $\overline{1}$ in the main diagonal and $E_{1}, \cdots, E_{r}$ being idempotents leads to the same problem, finding the maximum cardinality of $S_{i}, i \in\{1,2,3,4\}$. We finally conclude that 
$\left|S_{1}\right|=\left|S_{4}\right|=1$ and $\left|S_{2}\right|=\left|S_{3}\right|=p$, because the free variable $\alpha$ can take exactly the $p$ values $\overline{0}, \overline{1}, \ldots, \overline{p-1}$. So, $w n c\left(\mathbb{T}_{2}\left(\mathbb{Z}_{p}\right)\right)=p$, as promised.

Example 2.24. $w n c\left(\mathbb{T}_{3}\left(\mathbb{Z}_{p}\right)\right)=p^{2}$, where $p$ is a prime number.

Proof. It is a well-known fact that a matrix in $\mathbb{T}_{3}\left(\mathbb{Z}_{p}\right)$ is a nilpotent if and only if it has a zero main diagonal. We are looking now for idempotents. In fact, $\left(\begin{array}{ccc}x_{1} & a & b \\ \overline{0} & x_{2} & c \\ \overline{0} & \overline{0} & x_{3}\end{array}\right)\left(\begin{array}{ccc}x_{1} & a & b \\ \overline{0} & x_{2} & c \\ \overline{0} & \overline{0} & x_{3}\end{array}\right)=\left(\begin{array}{ccc}x_{1} & a & b \\ \overline{0} & x_{2} & c \\ \overline{0} & \overline{0} & x_{3}\end{array}\right)$, which is equivalent to $\left(\begin{array}{ccc}x_{1}^{2} & a\left(x_{1}+x_{2}\right) & b\left(x_{1}+x_{3}\right)+a c \\ \overline{0} & x_{2}^{2} & c\left(x_{2}+x_{3}\right) \\ \overline{0} & \overline{0} & x_{3}^{2}\end{array}\right)=\left(\begin{array}{ccc}x_{1} & a & b \\ \overline{0} & x_{2} & c \\ \overline{0} & \overline{0} & x_{3}\end{array}\right)$, which is equivalent to

$$
\left\{\begin{array}{l}
x_{1} \in\{\overline{0}, \overline{1}\} \\
x_{2} \in\{\overline{0}, \overline{1}\} \\
x_{3} \in\{\overline{0}, \overline{1}\} \\
a\left(x_{1}+x_{2}-\overline{1}\right)=\overline{0} \\
b\left(x_{1}+x_{3}-\overline{1}\right)=-a c \\
c\left(x_{2}+x_{3}-\overline{1}\right)=\overline{0}
\end{array}\right.
$$

For $x_{1}=\overline{0}, x_{2}=\overline{0}, x_{3}=\overline{0}$, we have $S_{1}=\left\{O_{3}\right\}$.

For $x_{1}=\overline{0}, x_{2}=\overline{0}, x_{3}=\overline{1}$, we have $S_{2}=\left\{\left(\begin{array}{ccc}\overline{0} & \overline{0} & \alpha \\ \overline{0} & \overline{0} & \gamma \\ \overline{0} & \overline{0} & \overline{1}\end{array}\right) \mid \alpha, \gamma \in \mathbb{Z}_{p}\right\}$.

For $x_{1}=\overline{0}, x_{2}=\overline{1}, x_{3}=\overline{0}$, we have $S_{3}=\left\{\left(\begin{array}{ccc}\overline{0} & \alpha & \alpha \gamma \\ \overline{0} & \overline{1} & \gamma \\ \overline{0} & \overline{0} & \overline{0}\end{array}\right) \mid \alpha, \gamma \in \mathbb{Z}_{p}\right\}$.

For $x_{1}=\overline{0}, x_{2}=\overline{1}, x_{3}=\overline{1}$, we have $S_{4}=\left\{\left(\begin{array}{ccc}\overline{0} & \alpha & \beta \\ \overline{0} & \overline{1} & \overline{0} \\ \overline{0} & \overline{0} & \overline{1}\end{array}\right) \mid \alpha, \beta \in \mathbb{Z}_{p}\right\}$.

For $x_{1}=\overline{1}, x_{2}=\overline{0}, x_{3}=\overline{0}$, we have $S_{5}=\left\{\left(\begin{array}{ccc}\overline{0} & \alpha & \beta \\ \overline{0} & \overline{0} & \overline{0} \\ \overline{0} & \overline{0} & \overline{0}\end{array}\right) \mid \alpha, \beta \in \mathbb{Z}_{p}\right\}$.

For $x_{1}=\overline{1}, x_{2}=\overline{0}, x_{3}=\overline{1}$, we have $S_{6}=\left\{\left(\begin{array}{ccc}\overline{1} & \alpha & -\alpha \gamma \\ \overline{0} & \overline{0} & \gamma \\ \overline{0} & \overline{0} & \overline{1}\end{array}\right) \mid \alpha, \gamma \in \mathbb{Z}_{p}\right\}$. 
For $x_{1}=\overline{1}, x_{2}=\overline{1}, x_{3}=\overline{0}$, we have $S_{7}=\left\{\left(\begin{array}{ccc}\overline{1} & \overline{0} & \alpha \\ \overline{0} & \overline{1} & \gamma \\ \overline{0} & \overline{0} & \overline{0}\end{array}\right) \mid \alpha, \gamma \in \mathbb{Z}_{p}\right\}$.

For $x_{1}=\overline{1}, x_{2}=\overline{1}, x_{3}=\overline{1}$, we have $S_{8}=\left\{I_{3}\right\}$. Following the same argument as in Example 2.23, we derive that wnc $\left(\mathbb{T}_{3}\left(\mathbb{Z}_{3}\right)\right)$ is the maximum cardinality of $S_{i}$, $i \in\{1,2, \ldots, 8\}$. Since $\left|S_{1}\right|=\left|S_{8}\right|=1$ and $\left|S_{2}\right|=\left|S_{3}\right|=\ldots=\left|S_{7}\right|=p^{2}$ (2 free variables and $\left.\left|\mathbb{Z}_{p}\right|=p\right)$, it finally follows that $w n c\left(\mathbb{T}_{3}\left(\mathbb{Z}_{p}\right)\right)=p^{2}$, as stated.

Remark 2.25. When studying weakly nil-clean matrices, it is not enough to study companion matrices which are (or are not) blocks of other companion matrices. In fact, note that not all matrices are similar to a companion matrix (see the proof of the main result in [2] or [4]).

Example 2.26. $w n c\left(\mathbb{M}_{2}\left(\mathbb{Z}_{3}\right)\right)=5$.

Proof. Let $A=\left(\begin{array}{ll}a & b \\ c & d\end{array}\right) \in \mathbb{M}_{2}\left(\mathbb{Z}_{3}\right)$. Then $A^{2}=\left(\begin{array}{cc}a^{2}+b c & b(a+d) \\ c(a+d) & d^{2}+b c\end{array}\right)$. We claim $A^{2}=A$ in order to find idempotents. They are the following:

$$
\left(\begin{array}{cc}
\overline{0} & s \\
\overline{0} & \overline{1}
\end{array}\right),\left(\begin{array}{cc}
\overline{1} & \overline{0} \\
s & 0
\end{array}\right),\left(\begin{array}{cc}
\overline{1} & \overline{0} \\
s & \overline{1}
\end{array}\right),\left(\begin{array}{cc}
\overline{0} & \overline{0} \\
s & \overline{1}
\end{array}\right),
$$

where $s \in \mathbb{Z}_{3}$ and also

$$
\left(\begin{array}{ll}
\overline{2} & \overline{2} \\
\overline{2} & \overline{2}
\end{array}\right),\left(\begin{array}{cc}
\overline{2} & \overline{1} \\
\overline{1} & \overline{2}
\end{array}\right)
$$

Next, we claim $A^{2}=\mathrm{O}_{2}$ to find out nilpotents. They are the following:

$$
\left(\begin{array}{ll}
\overline{1} & \overline{1} \\
\overline{2} & \overline{2}
\end{array}\right),\left(\begin{array}{cc}
\overline{2} & \overline{1} \\
\overline{2} & \overline{1}
\end{array}\right),\left(\begin{array}{cc}
\overline{2} & \overline{2} \\
\overline{1} & \overline{1}
\end{array}\right),\left(\begin{array}{cc}
\overline{1} & \overline{2} \\
\overline{1} & \overline{2}
\end{array}\right)
$$

and also

$$
\left(\begin{array}{cc}
\overline{0} & s \\
\overline{0} & \overline{0}
\end{array}\right),\left(\begin{array}{cc}
\overline{0} & \overline{0} \\
s & \overline{0}
\end{array}\right)
$$

where $s \in \mathbb{Z}_{3}$.

If $A+E$, with $E$ an idempotent, is nilpotent, then $\operatorname{tr}(A+E)=0$, whence $\operatorname{tr} A=-\operatorname{tr} E$. If $A-E$, with $E$ an idempotent, is nilpotent, then $\operatorname{tr}(A-E)=0$, whence $\operatorname{tr} A=\operatorname{tr} E$.

For an idempotent $E$, we deduce:

- $\operatorname{tr} E=\overline{1}$ if and only if $E \neq O_{2}, E \neq I_{2}$;

- $\operatorname{tr} E=\overline{2}$ if and only if $E=I_{2}$;

- $\operatorname{tr} E=\overline{0}$ if and only if $E=O_{2}$. 
Let $A=\left(\begin{array}{cc}\overline{0} & y \\ \overline{1} & \overline{0}\end{array}\right)$. Then, for an idempotent $E$, if $A+E$ is a nilpotent, then $\operatorname{tr}(E)=0$, and thus $E=O_{2}$. Also, if $A-E$ is a nilpotent, then $\operatorname{tr}(E)=0$ and hence $E=O_{2}$. Therefore, if $A=\left(\begin{array}{cc}\overline{0} & y \\ \overline{1} & \overline{0}\end{array}\right)$, we have $\alpha(A)=\left\{O_{2}\right\}$, and it follows that

$$
w n c\left(\left(\begin{array}{cc}
\overline{0} & y \\
\overline{1} & \overline{0}
\end{array}\right)\right) \leq 1
$$

such that $w n c\left(\left(\begin{array}{cc}\overline{0} & \overline{0} \\ \overline{1} & \overline{0}\end{array}\right)\right)=1$ and $w n c\left(\left(\begin{array}{cc}\overline{0} & \overline{1} \\ \overline{1} & \overline{0}\end{array}\right)\right)=w n c\left(\left(\begin{array}{cc}\overline{0} & \overline{2} \\ \overline{1} & \overline{0}\end{array}\right)\right)=0$.

Let $A=\left(\begin{array}{cc}\overline{0} & y \\ \overline{1} & \overline{1}\end{array}\right)$. Furthermore, for an idempotent $E$, if $A+E$ is a nilpotent, then $\operatorname{tr}(E)=\overline{2}$, and so $E=I_{2}$. But $\left(\begin{array}{cc}\overline{0} & y \\ \overline{1} & \overline{1}\end{array}\right)+\left(\begin{array}{cc}\overline{1} & \overline{0} \\ \overline{0} & \overline{1}\end{array}\right)=\left(\begin{array}{cc}\overline{1} & y \\ \overline{1} & \overline{2}\end{array}\right)$ is a nilpotent if and only if $y=\overline{2}$. Also, if $A-E$ is a nilpotent, then $\operatorname{tr}(E)=1$ and hence $E \neq O_{2}, I_{2}$.

We infer that

- $\left(\begin{array}{cc}\overline{0} & y \\ \overline{1} & \overline{1}\end{array}\right)-\left(\begin{array}{cc}\overline{0} & s \\ \overline{0} & \overline{1}\end{array}\right)=\left(\begin{array}{cc}\overline{0} & y-s \\ \overline{1} & \overline{0}\end{array}\right)$ is a nilpotent if and only if $s=y$;

- $\left(\begin{array}{ll}\overline{0} & y \\ \overline{1} & \overline{1}\end{array}\right)-\left(\begin{array}{ll}\overline{1} & \overline{0} \\ s & \overline{0}\end{array}\right)=\left(\begin{array}{cc}\overline{2} & y \\ \overline{1}-s & \overline{1}\end{array}\right)$ is a nilpotent if and only if $(y=\overline{2}$ and $s=\overline{0})$ or $(y=\overline{1}$ and $s=\overline{2})$;

- $\left(\begin{array}{cc}\overline{0} & y \\ \overline{1} & \overline{1}\end{array}\right)-\left(\begin{array}{cc}\overline{1} & s \\ \overline{0} & \overline{0}\end{array}\right)=\left(\begin{array}{cc}\overline{1} & y-s \\ \overline{1} & \overline{1}\end{array}\right)$, which is not a nilpotent;

- $\left(\begin{array}{ll}\overline{0} & y \\ \overline{1} & \overline{1}\end{array}\right)-\left(\begin{array}{ll}\overline{0} & \overline{0} \\ s & \overline{1}\end{array}\right)=\left(\begin{array}{cc}\overline{0} & y \\ \overline{1}-s & \overline{1}\end{array}\right)$, is a nilpotent if and only if $(y=\overline{0}$ and $\left.s \in \mathbb{Z}_{3}\right)$ or $\left(y \in \mathbb{Z}_{3}\right.$ and $\left.s=\overline{1}\right)$;

- $\left(\begin{array}{ll}\overline{0} & y \\ \overline{1} & \overline{1}\end{array}\right)-\left(\begin{array}{cc}\overline{2} & \overline{2} \\ \overline{2} & \overline{2}\end{array}\right)=\left(\begin{array}{cc}\overline{1} & y-\overline{2} \\ \overline{2} & \overline{2}\end{array}\right)$, which is a nilpotent if and only if $y=\overline{0}$;

- $\left(\begin{array}{ll}\overline{0} & y \\ \overline{1} & \overline{1}\end{array}\right)-\left(\begin{array}{cc}\overline{2} & \overline{1} \\ \overline{1} & \overline{2}\end{array}\right)=\left(\begin{array}{cc}\overline{2} & y-\overline{1} \\ \overline{0} & \overline{2}\end{array}\right)$, which is not a nilpotent.

By virtue of the above results, we get the following:

For $A=\left(\begin{array}{cc}\overline{0} & \overline{0} \\ \overline{1} & \overline{1}\end{array}\right)$ we have $E_{1}=\left(\begin{array}{cc}\overline{0} & s \\ \overline{0} & \overline{1}\end{array}\right), E_{2}=\left(\begin{array}{cc}\overline{0} & \overline{0} \\ \overline{0} & \overline{1}\end{array}\right), E_{3}=\left(\begin{array}{cc}\overline{0} & \overline{0} \\ \overline{1} & \overline{1}\end{array}\right)$, $E_{4}=\left(\begin{array}{cc}\overline{0} & \overline{0} \\ \overline{2} & \overline{1}\end{array}\right), E_{5}=\left(\begin{array}{cc}\overline{2} & \overline{2} \\ \overline{2} & \overline{2}\end{array}\right)$ such that $A-E_{i}$ is a nilpotent $(i \in\{1,2,3,4,5\})$ 
and there are no idempotents $E$ such that $A+E$ is a nilpotent. So

$$
\operatorname{wnc}\left(\left(\begin{array}{cc}
\overline{0} & \overline{0} \\
\overline{1} & \overline{1}
\end{array}\right)\right)=5 \text {. }
$$

For $A=\left(\begin{array}{cc}\overline{0} & \overline{1} \\ \overline{1} & \overline{1}\end{array}\right)$ we obtain the idempotents $E_{1}=\left(\begin{array}{cc}\overline{0} & \overline{1} \\ \overline{0} & \overline{1}\end{array}\right), E_{2}=\left(\begin{array}{cc}\overline{1} & \overline{0} \\ \overline{2} & \overline{0}\end{array}\right)$, $E_{3}=\left(\begin{array}{cc}\overline{0} & \overline{0} \\ \overline{1} & \overline{1}\end{array}\right)$ such that $A-E_{i}$ is a nilpotent, $i \in\{1,2,3\}$ and there are no idempotents $E$ such that $A+E$ is a nilpotent. So

$$
\text { wnc }\left(\left(\begin{array}{cc}
\overline{0} & \overline{1} \\
\overline{1} & \overline{1}
\end{array}\right)\right)=3 \text {. }
$$

For $A=\left(\begin{array}{cc}\overline{0} & \overline{2} \\ \overline{1} & \overline{1}\end{array}\right)$ we obtain the idempotents $E_{1}=\left(\begin{array}{cc}\overline{0} & \overline{2} \\ \overline{0} & \overline{1}\end{array}\right), E_{2}=\left(\begin{array}{cc}\overline{1} & \overline{0} \\ \overline{0} & \overline{0}\end{array}\right)$, $E_{3}=\left(\begin{array}{cc}\overline{0} & \overline{0} \\ \overline{1} & \overline{1}\end{array}\right)$ such that $A-E_{i}$ is a nilpotent, $i \in\{1,2,3\}$ and there is one idempotent, namely $E=I_{2}$ such that $A+E$ is a nilpotent. So

$$
\text { wnc }\left(\left(\begin{array}{cc}
\overline{0} & \overline{1} \\
\overline{1} & \overline{1}
\end{array}\right)\right)=4 \text {. }
$$

Let $A=\left(\begin{array}{cc}\overline{0} & y \\ \overline{1} & \overline{2}\end{array}\right)$. Furthermore, for an idempotent $E$, if $A+E$ is a nilpotent, then $\operatorname{tr}(E)=\overline{1}$, and thus $E \neq I_{2}, O_{2}$. Also, if $A-E$ is a nilpotent, then $\operatorname{tr}(E)=\overline{2}$ and hence $E=I_{2}$.

We derive

$\left(\begin{array}{cc}\overline{0} & y \\ \overline{1} & \overline{2}\end{array}\right)-\left(\begin{array}{cc}\overline{1} & \overline{0} \\ \overline{0} & \overline{1}\end{array}\right)=\left(\begin{array}{cc}\overline{2} & y \\ \overline{1} & \overline{1}\end{array}\right)$, which is a nilpotent if and only if $y=\overline{2}$

- Let $A=\left(\begin{array}{cc}\overline{0} & \overline{0} \\ \overline{1} & \overline{2}\end{array}\right)$. Then $\left(\begin{array}{cc}\overline{0} & \overline{0} \\ \overline{1} & \overline{2}\end{array}\right)+\left(\begin{array}{cc}\overline{0} & s \\ \overline{0} & \overline{1}\end{array}\right)=\left(\begin{array}{cc}\overline{0} & s \\ \overline{1} & \overline{0}\end{array}\right)$ is a nilpotent if and only if $s=0$;

- $\left(\begin{array}{cc}\overline{0} & \overline{1} \\ \overline{1} & \overline{2}\end{array}\right)+\left(\begin{array}{cc}\overline{1} & \overline{0} \\ s & \overline{0}\end{array}\right)=\left(\begin{array}{cc}\overline{1} & \overline{0} \\ \overline{1}+s & \overline{2}\end{array}\right)$, which is not a nilpotent;

- $\left(\begin{array}{cc}\overline{0} & \overline{0} \\ \overline{1} & \overline{2}\end{array}\right)+\left(\begin{array}{cc}\overline{1} & s \\ \overline{0} & \overline{0}\end{array}\right)=\left(\begin{array}{cc}\overline{1} & s \\ \overline{1} & \overline{2}\end{array}\right)$, which is a nilpotent if and only if $s=\overline{2}$

- $\left(\begin{array}{cc}\overline{0} & \overline{0} \\ \overline{1} & \overline{2}\end{array}\right)+\left(\begin{array}{cc}\overline{0} & \overline{0} \\ s & \overline{1}\end{array}\right)=\left(\begin{array}{cc}\overline{0} & \overline{0} \\ \overline{1}+s & \overline{0}\end{array}\right)$, is a nilpotent; 
- $\left(\begin{array}{cc}\overline{0} & \overline{0} \\ \overline{1} & \overline{2}\end{array}\right)+\left(\begin{array}{cc}\overline{2} & \overline{2} \\ \overline{2} & \overline{2}\end{array}\right)=\left(\begin{array}{cc}\overline{2} & \overline{2} \\ \overline{0} & \overline{1}\end{array}\right)$, which is not a nilpotent;

- $\left(\begin{array}{cc}\overline{0} & \overline{0} \\ \overline{1} & \overline{2}\end{array}\right)+\left(\begin{array}{cc}\overline{2} & \overline{1} \\ \overline{1} & \overline{2}\end{array}\right)=\left(\begin{array}{cc}\overline{2} & \overline{1} \\ \overline{2} & \overline{1}\end{array}\right)$, which is a nilpotent.

For $A=\left(\begin{array}{cc}\overline{0} & \overline{0} \\ \overline{1} & \overline{2}\end{array}\right)$ we have the idempotents $E_{1}=\left(\begin{array}{cc}\overline{0} & \overline{0} \\ \overline{0} & \overline{1}\end{array}\right), E_{2}=\left(\begin{array}{cc}\overline{1} & \overline{2} \\ \overline{0} & \overline{0}\end{array}\right)$, $E_{3}=\left(\begin{array}{cc}\overline{0} & \overline{0} \\ s & \overline{1}\end{array}\right), E_{4}=\left(\begin{array}{cc}\overline{2} & \overline{1} \\ \overline{1} & \overline{2}\end{array}\right)$ such that $A+E_{i}$ is a nilpotent, $i \in\{1,2,3,4\}$ and there are no idempotents $E$ such that $A-E$ is a nilpotent. So

$$
\text { wnc }\left(\left(\begin{array}{cc}
\overline{0} & \overline{0} \\
\overline{1} & \overline{2}
\end{array}\right)\right)=4 \text {. }
$$

Let $A=\left(\begin{array}{cc}\overline{0} & \overline{1} \\ \overline{1} & \overline{2}\end{array}\right)$.

- $\left(\begin{array}{cc}\overline{0} & \overline{1} \\ \overline{1} & \overline{2}\end{array}\right)+\left(\begin{array}{cc}\overline{0} & s \\ \overline{0} & \overline{1}\end{array}\right)=\left(\begin{array}{cc}\overline{0} & s+1 \\ \overline{1} & \overline{0}\end{array}\right)$ is a nilpotent if and only if $s=\overline{2}$;

- $\left(\begin{array}{cc}\overline{0} & \overline{1} \\ \overline{1} & \overline{2}\end{array}\right)+\left(\begin{array}{cc}\overline{1} & \overline{0} \\ s & \overline{0}\end{array}\right)=\left(\begin{array}{cc}\overline{1} & \overline{1} \\ \overline{1}+s & \overline{2}\end{array}\right)$, which is a nilpotent if and only if $s=\overline{1}$;

- $\left(\begin{array}{cc}\overline{0} & \overline{1} \\ \overline{1} & \overline{2}\end{array}\right)+\left(\begin{array}{cc}\overline{1} & s \\ \overline{0} & \overline{0}\end{array}\right)=\left(\begin{array}{cc}\overline{1} & s+1 \\ \overline{1} & \overline{2}\end{array}\right)$, which is a nilpotent if and only if $s=\overline{1}$;

- $\left(\begin{array}{cc}\overline{0} & \overline{1} \\ \overline{1} & \overline{2}\end{array}\right)+\left(\begin{array}{cc}\overline{0} & \overline{1} \\ s+1 & \overline{1}\end{array}\right)=\left(\begin{array}{cc}\overline{0} & \overline{1} \\ \overline{1}+s & \overline{0}\end{array}\right)$, is a nilpotent if and only if $s=\overline{2}$

- $\left(\begin{array}{cc}\overline{0} & \overline{1} \\ \overline{1} & \overline{2}\end{array}\right)+\left(\begin{array}{cc}\overline{2} & \overline{2} \\ \overline{2} & \overline{2}\end{array}\right)=\left(\begin{array}{cc}\overline{2} & \overline{0} \\ \overline{0} & \overline{1}\end{array}\right)$, which is not a nilpotent;

- $\left(\begin{array}{cc}\overline{0} & \overline{1} \\ \overline{1} & \overline{2}\end{array}\right)+\left(\begin{array}{cc}\overline{2} & \overline{1} \\ \overline{1} & \overline{2}\end{array}\right)=\left(\begin{array}{cc}\overline{2} & \overline{2} \\ \overline{2} & \overline{1}\end{array}\right)$, which is not a nilpotent.

For $A=\left(\begin{array}{cc}\overline{0} & \overline{1} \\ \overline{1} & \overline{2}\end{array}\right)$ we obtain the idempotents $E_{1}=\left(\begin{array}{cc}\overline{0} & \overline{2} \\ \overline{0} & \overline{1}\end{array}\right), E_{2}=\left(\begin{array}{cc}\overline{1} & \overline{0} \\ \overline{1} & \overline{0}\end{array}\right)$, $E_{3}=\left(\begin{array}{cc}\overline{1} & \overline{1} \\ \overline{0} & \overline{0}\end{array}\right), E_{4}=\left(\begin{array}{cc}\overline{0} & \overline{0} \\ \overline{2} & \overline{1}\end{array}\right)$ such that $A+E_{i}$ is a nilpotent, $i \in\{1,2,3,4\}$ and there are no idempotents $E$ such that $A-E$ is a nilpotent. So

$$
\operatorname{wnc}\left(\left(\begin{array}{cc}
\overline{0} & \overline{1} \\
\overline{1} & \overline{2}
\end{array}\right)\right)=4 \text {. }
$$


Let $A=\left(\begin{array}{cc}\overline{0} & \overline{2} \\ \overline{1} & \overline{2}\end{array}\right)$. We have

- $\left(\begin{array}{cc}\overline{0} & \overline{2} \\ \overline{1} & \overline{2}\end{array}\right)+\left(\begin{array}{cc}\overline{0} & s \\ \overline{0} & \overline{1}\end{array}\right)=\left(\begin{array}{cc}\overline{0} & s+\overline{2} \\ \overline{1} & \overline{0}\end{array}\right)$ is a nilpotent if and only if $s=\overline{1}$;

- $\left(\begin{array}{cc}\overline{0} & \overline{2} \\ \overline{1} & \overline{2}\end{array}\right)+\left(\begin{array}{cc}\overline{1} & \overline{0} \\ s & \overline{0}\end{array}\right)=\left(\begin{array}{cc}\overline{1} & \overline{2} \\ \overline{1}+s & \overline{2}\end{array}\right)$, which is a nilpotent if and only if $s=\overline{1}$.

- $\left(\begin{array}{cc}\overline{0} & \overline{2} \\ \overline{1} & \overline{2}\end{array}\right)+\left(\begin{array}{cc}\overline{1} & s \\ \overline{0} & \overline{0}\end{array}\right)=\left(\begin{array}{cc}\overline{1} & s+\overline{2} \\ \overline{1} & \overline{2}\end{array}\right)$, which is a nilpotent if and only if $s=\overline{0}$;

- $\left(\begin{array}{cc}\overline{0} & \overline{2} \\ \overline{1} & \overline{2}\end{array}\right)+\left(\begin{array}{cc}\overline{0} & \overline{1} \\ s+1 & \overline{1}\end{array}\right)=\left(\begin{array}{cc}\overline{0} & \overline{2} \\ \overline{1}+s & \overline{0}\end{array}\right)$, is a nilpotent if and only if $s=\overline{2}$

- $\left(\begin{array}{cc}\overline{0} & \overline{2} \\ \overline{1} & \overline{2}\end{array}\right)+\left(\begin{array}{cc}\overline{2} & \overline{2} \\ \overline{2} & \overline{2}\end{array}\right)=\left(\begin{array}{cc}\overline{2} & \overline{1} \\ \overline{0} & \overline{1}\end{array}\right)$, which is not a nilpotent;

- $\left(\begin{array}{cc}\overline{0} & \overline{2} \\ \overline{1} & \overline{2}\end{array}\right)+\left(\begin{array}{cc}\overline{2} & \overline{1} \\ \overline{1} & \overline{2}\end{array}\right)=\left(\begin{array}{cc}\overline{2} & \overline{0} \\ \overline{2} & \overline{1}\end{array}\right)$, which is not a nilpotent.

For $A=\left(\begin{array}{cc}\overline{0} & \overline{2} \\ \overline{1} & \overline{2}\end{array}\right)$ we obtain the idempotents $E_{1}=\left(\begin{array}{cc}\overline{0} & \overline{1} \\ \overline{0} & \overline{1}\end{array}\right), E_{2}=\left(\begin{array}{cc}\overline{1} & \overline{0} \\ \overline{0} & \overline{1}\end{array}\right)$, $E_{3}=\left(\begin{array}{cc}\overline{1} & \overline{0} \\ \overline{0} & \overline{0}\end{array}\right), E_{4}=\left(\begin{array}{cc}\overline{0} & \overline{0} \\ \overline{2} & \overline{2}\end{array}\right)$ such that $A+E_{i}$ is a nilpotent, $i \in\{1,2,3,4\}$ and there is one idempotent $E=I_{2}$ such that $A-E$ is a nilpotent. So

$$
\text { wnc }\left(\left(\begin{array}{cc}
\overline{0} & \overline{1} \\
\overline{1} & \overline{2}
\end{array}\right)\right)=5 \text {. }
$$

In conclusion, $\operatorname{wnc}\left(\mathbb{M}_{2}\left(\mathbb{Z}_{3}\right)\right)=5$, as expected.

For rings $A$ and $B$ and for a bimodule ${ }_{A} M_{B}$, we denote by $\left(\begin{array}{cc}A & M \\ 0 & B\end{array}\right)$ the formal triangular matrix ring.

The next statement strengthens [1, Theorem 4.1].

Proposition 2.27. Let $R$ be a ring. The following statements are equivalent:

(1) $\operatorname{wnc}(R)=2$;

(2) $R=\left(\begin{array}{cc}A & M \\ 0 & B\end{array}\right)$, where $A$ and $B$ are abelian rings, and ${ }_{A} M_{B}$ is a bimodule with $|M|=2$. 
Proof. $(1) \Rightarrow(2)$ :

If $\operatorname{wnc}(R)=2$, since $\operatorname{wnc}(R) \geq \operatorname{Nin}(R)$, then $\operatorname{Nin}(R)=1$ or $\operatorname{Nin}(R)=2$.

- If $\operatorname{Nin}(R)=1$, then $R$ is abelian and so $\operatorname{wnc}(R)=1$, which is a contradiction.

- If $\operatorname{Nin}(R)=2$, then by Theorem 4.1 in [1] we get the desired form of $R$.

$(2) \Rightarrow(1)$ :

Nilpotent elements in $R$ are $\left(\begin{array}{cc}n_{A} & w \\ 0 & n_{B}\end{array}\right)$, where $n_{A}$ is a nilpotent in $A, n_{B}$ is a nilpotent in $B$ and $w$ is any element in $M=\{0, x\}$.

Idempotent elements in $R$ are $\left(\begin{array}{cc}e_{A} & w \\ 0 & e_{B}\end{array}\right)$, where $e_{A}$ is an idempotent in $A, e_{B}$ is an idempotent in $B$ and $w \in M$ which satisfies the condition $e_{A} w+w e_{B}=w$. Since $\operatorname{wnc}(A)=\operatorname{wnc}(B)=1$ and $x=x+0=0+x=x-0=0-x$ are the only decompositions of $x$, we have at most four weakly nil clean decompositions for $\left(\begin{array}{ll}a & x \\ 0 & b\end{array}\right)$ as follows:

$$
\begin{gathered}
\left(\begin{array}{cc}
a & w \\
0 & b
\end{array}\right)=\left(\begin{array}{cc}
n_{A} & x \\
0 & n_{B}
\end{array}\right)+\left(\begin{array}{cc}
e_{A} & 0 \\
0 & e_{B}
\end{array}\right) ; \\
\left(\begin{array}{ll}
a & x \\
0 & b
\end{array}\right)=\left(\begin{array}{cc}
n_{A} & 0 \\
0 & n_{B}
\end{array}\right)+\left(\begin{array}{cc}
e_{A} & x \\
0 & e_{B}
\end{array}\right) \text { with } e_{A} x+x e_{B}=0 ; \\
\left(\begin{array}{cc}
a & x \\
0 & b
\end{array}\right)=\left(\begin{array}{cc}
n_{A}^{\prime} & x \\
0 & n_{B}^{\prime}
\end{array}\right)-\left(\begin{array}{cc}
e_{A} & 0 \\
0 & e_{B}
\end{array}\right) ; \\
\left(\begin{array}{ll}
a & x \\
0 & b
\end{array}\right)=\left(\begin{array}{cc}
n_{A}^{\prime} & 0 \\
0 & n_{B}^{\prime}
\end{array}\right)-\left(\begin{array}{cc}
e_{A} & x \\
0 & e_{B}
\end{array}\right) \text { with } e_{A} x+x e_{B}=x .
\end{gathered}
$$

Hence we get at most two idempotents in $\alpha\left(\left(\begin{array}{cc}a & x \\ 0 & b\end{array}\right)\right)$.

Since $\operatorname{wnc}(A)=w n c(B)=1$ and $0=0+0=x+x=0-0=x-x$ are the only decompositions of $x$, we have at most four weakly nil clean decompositions for $\left(\begin{array}{ll}a & 0 \\ 0 & b\end{array}\right)$ as follows:

$$
\left(\begin{array}{ll}
a & 0 \\
0 & b
\end{array}\right)=\left(\begin{array}{cc}
n_{A} & 0 \\
0 & n_{B}
\end{array}\right)+\left(\begin{array}{cc}
e_{A} & 0 \\
0 & e_{B}
\end{array}\right) ;
$$




$$
\begin{gathered}
\left(\begin{array}{ll}
a & 0 \\
0 & b
\end{array}\right)=\left(\begin{array}{cc}
n_{A} & x \\
0 & n_{B}
\end{array}\right)+\left(\begin{array}{cc}
e_{A} & x \\
0 & e_{B}
\end{array}\right) \text { with } e_{A} x+x e_{B}=x ; \\
\left(\begin{array}{ll}
a & 0 \\
0 & b
\end{array}\right)=\left(\begin{array}{cc}
n_{A}^{\prime} & 0 \\
0 & n_{B}^{\prime}
\end{array}\right)-\left(\begin{array}{cc}
e_{A} & 0 \\
0 & e_{B}
\end{array}\right) ; \\
\left(\begin{array}{ll}
a & 0 \\
0 & b
\end{array}\right)=\left(\begin{array}{cc}
n_{A}^{\prime} & x \\
0 & n_{B}^{\prime}
\end{array}\right)-\left(\begin{array}{cc}
e_{A} & x \\
0 & e_{B}
\end{array}\right) \text { with } e_{A} x+x e_{B}=x .
\end{gathered}
$$

Hence we got at most 2 idempotents in $\alpha\left(\left(\begin{array}{ll}a & 0 \\ 0 & b\end{array}\right)\right)$.

Therefore, $w n c(R) \leq 2$, and so if we find $q$ in $R$ such that we can get two idempotents in $\alpha(q)$, then $w n c(R)=2$. Thus $q$ is $\left(\begin{array}{cc}0 & 0 \\ 0 & 1_{B}\end{array}\right)$ and the idempotents $\operatorname{are}\left(\begin{array}{cc}0 & 0 \\ 0 & 1_{B}\end{array}\right)$ and $\left(\begin{array}{cc}0 & x \\ 0 & 1_{B}\end{array}\right)$.

We continue by showing that the next assertion is not an analogue of [1, Proposition 4.2].

Example 2.28. If $R=\left(\begin{array}{cc}A & M \\ 0 & B\end{array}\right)$, where $w n c(A)=w n c(B)=1$ and ${ }_{A} M_{B}$ is a bimodule with $|M|=3$, then wnc $(R)=3$ cannot be happen in general. In fact, in accordance with Example 2.26, $R=\left(\begin{array}{cc}\mathbb{Z}_{3} & \mathbb{Z}_{3} \\ \mathbb{Z}_{3} & \mathbb{Z}_{3}\end{array}\right)=\left(\begin{array}{cc}\mathbb{Z}_{3} & \mathbb{Z}_{3} \\ 0 & \mathbb{Z}_{3}\end{array}\right)+\left(\begin{array}{cc}0 & 0 \\ \mathbb{Z}_{3} & 0\end{array}\right)$ is a ring with wnc $(R)=5>3$.

Note that if $P=\left(\begin{array}{cc}\mathbb{Z}_{3} & \mathbb{Z}_{3} \\ 0 & \mathbb{Z}_{3}\end{array}\right)$, then $P / J(P) \cong \mathbb{Z}_{3} \times \mathbb{Z}_{3}$.

We now proceed by extending [1, Proposition 4.4] in the following manner.

Proposition 2.29. Let $R$ be a ring and let $n \geq 1$ be an integer. Then

(i) $\operatorname{wnc}\left(\mathbb{M}_{n}(R)\right) \geq 3$, provided $n \geq 2$.

(ii) $w n c\left(\mathbb{M}_{n}(R)\right)=3$ if and only if $n=2$ and $R \cong \mathbb{Z}_{2}$.

Proof. (i) Applying Lemma 2.9, it follows that wnc $\left(\mathbb{M}_{n}(R)\right) \geq \operatorname{Nin}\left(\mathbb{M}_{n}(R)\right)$. Furthermore, [1, Proposition $4.4(1)]$ applies to get the wanted inequality.

(ii) Referring again to Lemma $2.9, \operatorname{Nin}\left(\mathbb{M}_{n}(R)\right) \leq w n c\left(\mathbb{M}_{n}(R)\right)$ so that either $\operatorname{Nin}\left(\mathbb{M}_{n}(R)\right)=1$, or $\operatorname{Nin}\left(\mathbb{M}_{n}(R)\right)=2$, or $\operatorname{Nin}\left(\mathbb{M}_{n}(R)\right)=3$. The first two cases are impossible appealing to [1, Theorem 3.2] or to [1, Theorem 4.1], respectively. The third case is handled in [1, Proposition 4.4 (2)], which gives our claim. 
Remark 2.30. It is noteworthy that by virtue of $[2]$ the ring $\mathbb{M}_{2}(R) \cong \mathbb{M}_{2}\left(\mathbb{Z}_{2}\right)$ is nil-clean and consequently wnc $\left(\mathbb{M}_{2}\left(\mathbb{Z}_{2}\right)\right)=\operatorname{Nin}\left(\mathbb{M}_{2}\left(\mathbb{Z}_{2}\right)\right)$.

\section{Open questions}

We finish the paper with a series of left-answered problems:

Problem 1. For a ring $R$ find a criterion when the equality $c(R)=w n c(R)$ holds.

Problem 2. For a ring $R$ find a criterion when the equality $c(R)=\operatorname{Nin}(R)$ holds.

Problem 3. For a ring $R$ find a criterion when the equality $w n c(R)=\operatorname{Nin}(R)$ holds.

Problem 4. If $R=S \times T$ is a direct decomposition of a ring $R$, does it follow that $w n c(R)=\operatorname{Nin}(S) w n c(T)=w n c(S) N i n(T)$ ?

In that direction, this is related to the existence of such rings $R$ satisfying the inequality $\operatorname{wnc}(R)>\operatorname{Nin}(R)$.

Acknowledgements. The authors are very indebted to the referee for his/her useful suggestions as well as to the editor, Professor Sait Halıcıŏlu, for his professional managing of the article. We are also deeply grateful to Professor Simion Breaz for his expert comments and encouragement.

\section{References}

[1] D. K. Basnet and J. Bhattacharyya, Nil clean index of rings, Int. Electron. J. Algebra, 15 (2014), 145-156.

[2] S. Breaz, G. Călugăreanu, P. Danchev and T. Micu, Nil-clean matrix rings, Linear Algebra Appl., 439(10) (2013), 3115-3119.

[3] S. Breaz, P. Danchev and Y. Zhou, Rings in which every element is either a sum or a difference of a nilpotent and an idempotent, J. Algebra Appl., 15(8) (2016), 1650148, 11 pp.

[4] S. Breaz and G. C. Modoi, Nil-clean companion matrices, Linear Algebra Appl., 489 (2016), 50-60.

[5] H. Chen, On uniquely clean rings, Comm. Algebra, 39(1) (2011), 189-198.

[6] P. V. Danchev, Weakly UU rings, Tsukuba J. Math., 40(1) (2016), 101-118.

[7] P. V. Danchev and W. Wm. McGovern, Commutative weakly nil clean unital rings, J. Algebra, 425 (2015), 410-422.

[8] A. J. Diesl, Nil clean rings, J. Algebra, 383 (2013), 197-211.

[9] T. Y. Lam, A First Course in Noncommutative Rings, Second Edition, Grad. Texts in Math., 131, Springer-Verlag, New York, 2001. 
[10] T. K. Lee and Y. Zhou, Clean index of rings, Comm. Algebra, 40(3) (2012), 807-822.

[11] T. K. Lee and Y. Zhou, Rings of clean index 4 and applications, Comm. Algebra, 41(1) (2013), 238-259.

[12] J. Šter, Nil clean involutions, arXiv preprint, (2015).

Andrada Cîmpean (Corresponding Author)

Faculty of Mathematics and Computer Science

Babeş-Bolyai University

400084 Cluj-Napoca, Romania

e-mail: cimpean_andrada@yahoo.com

Peter Danchev

Department of Mathematics

Plovdiv University

4000 Plovdiv, Bulgaria

e-mail:pvdanchev@yahoo.com 